\title{
Distinguishing short-term memory from working memory
}

\author{
ROBERT KAIL \\ University of Maryland, College Park, Maryland \\ and \\ LYNDA K. HALL \\ Ohio Wesleyan University, Delaware, Ohio
}

\begin{abstract}
The aim of the present research was to determine whether short-term memory and working memory could be distinguished. In two studies, 7 - to 13 -year-olds $(N=155, N=132)$ were administered tasks thought to assess short-term memory as well as tasks thought to assess working memory. Both exploratory and confirmatory factor analyses distinguished short-term memory tasks from working memory tasks. In addition, performance on working memory tasks was related to word decoding skill but performance on short-term memory tasks was not. Finally, performance on both short-term memory and working memory tasks were associated with age-relatedincreases in processing speed. Results are discussed in relation to models of short-term and working memory.
\end{abstract}

Short-term memory (STM) and working memory (WM) are both central constructs in modern theories of memory and cognition, but only recently have researchers begun to examine the relation between them. In a theory proposed by Cowan $(1988,1995)$, STM refers to information in long-term memory that is activated above some threshold. Activated information rapidly returns to an inactive state unless it becomes the focus of limited-capacity attentional processes. WM includes STM as well as the attentional processes used to keep some STM contents in an activated state. Similarly, Engle, Kane, and Tuholski (1999) argued that WM is "a system consisting of (a) a store in the form of long-term memory traces active above threshold, (b) processes for achieving and maintaining that activation, and (c) controlled attention" ( p. 104). Thus, in both theories, STM is a subcomponent of WM.

This theoretical account of the relation between STM and WM leads to two straightforward predictions. First, tasks that measure STM should be distinguishable from, but related to, tasks that measure WM. That is, because STM is a subset of WM, (i.e., $\mathrm{WM}=\mathrm{STM}+$ attention), performance on STM tasks should be related to performance on WM tasks. However, these correlations should be smaller than (1) correlations between performance on

The research described in this article was supported by a grant from the National Science Foundation (SBR-9413019). We thank Christina Conrad, René Kline, and Laura Curry for testing children; the staff and students of Otterbein Elementary School for their friendly cooperation; and Andy Conway for his help with the data analyses. Correspondence concerning this article should be sent to R. Kail, Department of Psychological Sciences, Purdue University, West Lafayette, IN 47907-1364 (e-mail: rkail@sla.purdue.edu). different STM tasks, and (2) correlations between performance on WM tasks. Of course, no tasks are pure measures of either STM or WM. Tasks thought to measure STM and WM often share other processes (e.g., encoding perceptual information, responding auditorily). These shared processes would tend to increase the size of the correlations between tasks. Consequently, the key prediction is that correlations measuring the same element of memory (STM or WM) should be greater than correlations measuring different elements (STM with WM).

The second prediction concerns links between STM, WM, and higher order cognitive constructs such as reasoning, problem solving, and reading. In the course of reasoning, solving problems, and reading, individuals often must remember some task elements and ignore or inhibit other elements as they complete task-relevant operations. That is, in terms of the theories described previously, attention is used to maintain task-relevant information in an active state and to regulate controlled processing. Consequently, the expectation is that although STM and WM should both be related to measures of problem solving and reading skill, performance on these tasks should be predicted more accurately by WM than by STM.

Regarding the first prediction, supporting evidence comes from two studies by Engle and his colleagues (Cantor, Engle, \& Hamilton, 1991; Engle, Tuholski, Laughlin, \& Conway, 1999). Simple digit span and word span tasks were administered, along with complex span tasks like those devised by Daneman and Carpenter (1980), in which participants read sets of sentences and concurrently remembered the last word from each sentence in the set. Factor analyses revealed that the simple span tasks and reading span tasks loaded on separate factors 
that were interpreted to reflect STM and WM, respectively. The factors were not independent but were strongly correlated.

Regarding the second prediction, many investigators have linked memory processes to reasoning, problem solving, and reading. Simple span tasks thought to assess STM are related to performance on a variety of complex cognitive tasks (e.g., Bachelder \& Denny, 1977) as are complex span tasks thought to assess working memory (e.g., Carpenter, Just, \& Shell, 1990; Kyllonen \& Christal, 1990). However, relatively few studies have compared the extent to which STM and WM predict performance on cognitive tasks. In the studies by Engle and his colleagues described previously (Cantor et al., 1991; Engle, Tuholski, et al., 1999), STM and WM both accounted for unique variance in verbal scores on the Scholastic Aptitude Test (SAT). In contrast, only WM accounted for significant variance in SAT quantitative scores and in measures of fluid intelligence such as Raven's Progressive Matrices. In addition, Swanson (1992) reported that 5- to 18 -year-olds' scores on the Reading Recognition scale of the Peabody Individual Achievement Test-Revised (PIAT-R) were more highly correlated with WM scores than with STM scores; however, this difference is difficult to interpret because WM was estimated with composites derived from multiple tasks, whereas STM was estimated with raw scores on individual tasks.

Thus, the existing evidence, though scanty, is consistent with the hypotheses that WM and STM are distinct but related and that WM plays a greater role than STM in higher order cognitive processes. The present research was designed to provide additional evidence concerning the distinction between WM and STM. The studies reported here followed much the same logic as the study by Engle, Tuholski, et al. (1999): Participants were administered simple and complex span tasks as well as tasks assessing other cognitive processes. If simple and complex span tasks represent STM and WM, respectively, these tasks should define different factors in factor analyses. If, instead, simple and complex span tasks assess a common, undifferentiated STM construct, these tasks should define a single, common factor. Furthermore, if WM has greater influence than that of STM on higher order cognitive processes, then higher order cognitive processes should be linked more strongly to complex span tasks than to simple span tasks.

The present work differed from that of Engle, Tuholski, et al. (1999) in three ways designed to provide converging evidence on the relation between STM and WM. First, the participants in the Engle, Tuholski, et al. study were undergraduates; the present studies included elementary school children ranging in age from 7 to 13 years. Thus, the present study allowed us to investigate STM and WM with a more heterogeneous sample (in both age and ability) than did Engle, Tuholski, et al. and allowed us to determine whether results for adults would extend to children. Second, we extended the range of tasks used to identify STM and WM constructs. In addition to Engle, Tuholski, et al.'s word and reading span tasks, we included letter and digit span tasks to assess STM as well as listening and least number span (in which participants identified, then remembered, the smallest of a set of three numbers) to assess WM. Third, rather than using scores on aptitude measures like the SAT or the Raven's to distinguish WM from STM, we used scores on a measure of word decoding. As noted previously, Swanson (1992) presented preliminary evidence indicating that word decoding was linked more strongly to WM than to STM. In the present work, children were administered a measure of word decoding skill so that we could evaluate links from STM and WM to word decoding.

An additional aim of the present research was to examine links from speed of information processing to both STM and WM. Many investigators have shown that processing speed (PS) is correlated positively with a variety of memory measures - more rapid processing is associated with better retention - and this result holds for children as well as younger and older adults (Cowan et al., 1998; Kail \& Park, 1994; Salthouse, 1996; Towse, Hitch, \& Hutton, 1998). However, the memory tasks used in these studies were not selected with the specific intent of comparing the contributions of PS to STM and WM, which was the aim here.

Two studies with 7- to 13-year-olds are reported. In each study, children were tested on STM, WM, PS, and word decoding tasks. Study 1 represents additional analyses of data reported by Kail and Hall (1999, Study 2). Study 2 has not been reported previously.

\section{METHOD}

\section{Participants}

Study 1 included 155 children, 29 eight-year-olds, 36 nine-yearolds, 36 ten-year-olds, 36 eleven-year-olds, and 18 twelve-yearolds. Study 2 included 142 children, 26 at each of 8-12 years of age and 12 at 13 years. All samples included approximately equal numbers of males and females. The children lived in various cities and towns in the midwestern United States.

\section{Tasks}

Memory. STM was assessed with traditional span tasks in which children remembered digits, letters, or words. For digit span, two digits were read aloud, at a rate of approximately one digit per second. After presentation of the last digit, the child attempted to recall the digits in the correct sequence. If recall was entirely correct, the procedure was repeated with another set of two digits. If the second recall was also correct, the number of digits was increased by one. This procedure was repeated until the child recalled a set of digits inaccurately. Digit span was defined as the largest set of digits for which the child recalled both sequences perfectly.

Procedures for letter and word span were the same, with nine letters or nine one-syllable words replacing, respectively, the digits 1-9.

WM was assessed with three tasks in which children remembered information while concurrently performing other cognitive tasks. Reading span, based on the task devised originally by Daneman and Carpenter (1980), included 5- to 10-word sentences that ended in a noun and made assertions that were obviously true ("A giraffe has a long neck") or false ("A fire is very cold"). The sentences were arbitrarily grouped to create sets containing one, two, three, four, and five sentences. Each sentence was printed on a separate card. The children were asked to (1) read aloud a sentence, (2) say whether the statement was true or false, (3) remember the 
final word in each sentence, and (4) turn the card and read the sentence on the next card. This was repeated until the children reached a card with a question mark, which was a signal to recall the final word from each sentence, in order. Two practice trials with onesentence sets were followed by the test trials, which began with onesentence sets and continued until the children failed to recall the words accurately or had completed both five-sentence sets. Reading span was defined as the largest set for which the children recalled all words correctly from both sentence sets.

Listening span was analogous to reading span, except that the children listened to sentences (presented via audio tape) instead of reading them. The sentences were constructed according to the same procedures as those for reading span and were recorded by an adult female speaking at a normal conversational pace. Listening span was defined as the largest set for which children recalled words from both sets of sentences.

In least number span, the children were shown a card with three numbers appearing on it: a number between 1 and 22 , a number between 23 and 44, and a number between 45 and 66. The three numbers appeared in a row on each card and, across cards, small, medium, and large numbers appeared equally often as the first, second, and third numbers in a row. The children first read the three numbers aloud, then named the smallest number. Another, similarly organized card was then presented, and the children again read aloud all three numbers, then stated the smallest one. Finally, a card with two blanks was shown and the children were asked to remember the smallest number from each card, in order. Testing began with two practice trials, each with two cards (thus two numbers to remember); then the children were administered two test trials of two cards, two test trials of three cards, and so on, until they incorrectly recalled the sequence of smallest numbers. Least number span was defined as the largest set of cards for which the children recalled the smallest numbers in order from both sets of cards.

Decoding skill. The Reading Recognition task of the PIAT-R was administered, following standard procedures in which the children read individual words aloud.

Processing speed. Two tasks from the Woodcock-Johnson Tests of Cognitive Ability - Visual Matching and Cross-Out-were used to estimate processing speed. In Visual Matching, each of 60 rows includes six digits, two of which are identical (e.g., 895297 ); the child circled the identical digits. The performance measure is the number of rows completed correctly in $3 \mathrm{~min}$. In the Cross-Out task, each of 30 rows consists of a geometric figure at the left end of a row and 19 similar figures to the right. One row, for example, consists of a triangle enclosing a single dot; the 19 figures are triangles with various objects in the interior (e.g., a single dot, three dots, a plus, a square). The child placed a line through the 5 figures of the 19 that are identical to the one at the left. The performance measure is the number of rows completed in $3 \mathrm{~min}$.

\section{Procedure}

Testing took place in quiet rooms in the children's school or in the authors' campus laboratories. Word span, reading span, listening span, word decoding, and PS tasks were administered in both studies; letter span was administered only in Study 1; digit span and least number span were administered only in Study 2. Tasks were presented in a single session, in a constant order. Order of tasks was not counterbalanced across children, so that individual differences would not be confounded with differences in task order. The presentation order for Study 1 was as follows: letter span, arithmetic word problems (not analyzed here), Visual Matching, reading span, addition and subtraction problems (not analyzed here), word span, arithmetic word problems, Cross-Out, letter span, addition and subtraction problems, and Reading Recognition. The presentation order for Study 2 was the following: reading span, Visual Matching, least number span, word span, Cross-Out, digit span, listening span, and Reading Recognition.

\section{RESULTS}

The results will be described in three sections: (1) relations between memory tasks, (2) links between memory tasks and decoding skill, and (3) links from PS to memory tasks.

\section{Relations Between Memory Tasks}

Shown in Table 1 are correlations between the 10 measures, separately for the two studies. All measures were correlated positively. Correlations between memory measures thought to reflect the same underlying construct (either STM or WM) are shown in bold in Table 1; correlations between tasks thought to measure different memory constructs are shown in italics. The former correlations tended to be larger than the latter correlations. Specifically, the $5 r$ s from tasks involving the same memory construct ranged from .36 to .47 , with a median of .45 ; the $10 r \mathrm{~s}$ from tasks involving different memory constructs ranged from .19 to .32 , with a median of .26 .

These patterns of correlations were evaluated with two sets of factor analyses. The first, an exploratory factor analysis, was designed to determine whether the memory tasks would load on two distinct factors. For these analyses, the memory tasks from each study were subjected to principal-components analysis with varimax rotation. The results, shown in Table 2, are consistent with the hypothesis that tasks thought to measure STM load on one factor, whereas tasks thought to measure WM load on another factor. That is, in each study, digit, letter, and word span tasks loaded on different factors from those for the reading, listening, and least-number span tasks.

After the exploratory factor analyses, we conducted confirmatory factor analyses in which the aim was to contrast two models. A two-factor model assumed that digit, letter, and word span tasks reflect STM, whereas reading, listening, and least number span tasks reflect WM. A one-factor model assumed that all the memory tasks used here reflect a single memory construct. Structural equation modeling was used to determine the fit of observed pattern of correlations to the structural relations specified in each model. The critical test involved comparing the fit of the two models. If the memory tasks reflect two distinct underlying constructs, then the twofactor model should fit the data significantly better than the one-factor model.

The fit of both models to the data was evaluated with EzPath (Steiger, 1989), using maximum likelihood estimation to derive best-fitting path coefficients. Several measures were used to evaluate the fit of the models to the data. The most familiar, $\chi^{2}$, was used to determine whether the data deviated from the model in a statistically significant manner. The Jöreskog-Sörbom goodness of fit index (GFI) ranges from 0 to 1 , with the latter indicating perfect correspondence between model and data; values greater than .90 indicate a good fit. The adjusted root mean square (ARMS) is a measure of the size of the residuals, adjusted for the complexity of the model; values 
Table 1

Correlations Between Measures in Studies 1 and 2

\begin{tabular}{|c|c|c|c|c|c|c|c|c|c|c|}
\hline Measure & Age & $\begin{array}{l}\text { Digit } \\
\text { Span }\end{array}$ & $\begin{array}{r}\text { Letter } \\
\text { Span }\end{array}$ & $\begin{array}{l}\text { Word } \\
\text { Span }\end{array}$ & $\begin{array}{l}\text { Read } \\
\text { Span }\end{array}$ & $\begin{array}{c}\text { Listen } \\
\text { Span }\end{array}$ & $\begin{array}{l}\text { Least } \\
\text { Span }\end{array}$ & $\mathrm{VM}$ & Cross Out & PIAT Rec \\
\hline \multicolumn{11}{|c|}{ Study $1(N=155)$} \\
\hline Letter span & .34 & - & & & & & & & & \\
\hline Word span & .18 & - & .47 & - & & & & & & \\
\hline Read span & .41 & - & .30 & .27 & - & & & & & \\
\hline Listen span & .34 & - & .26 & .24 & .45 & - & & & & \\
\hline VM & .63 & - & .28 & .25 & .32 & .37 & - & - & & \\
\hline Cross Out & .64 & - & .34 & .32 & .34 & .34 & - & .76 & - & \\
\hline PIAT Rec & .50 & - & .31 & .32 & .44 & .37 & - & .48 & .44 & - \\
\hline$M$ & 10.2 & - & 4.72 & 4.02 & 1.72 & 1.99 & - & 37.62 & 19.64 & 60.81 \\
\hline$S D$ & 1.21 & - & 0.84 & 0.52 & 0.52 & 0.60 & - & 5.84 & 3.94 & 14.53 \\
\hline \multicolumn{11}{|c|}{ Study $2(N=142)$} \\
\hline Digit span & .23 & - & & & & & & & & \\
\hline Word span & .19 & .37 & - & - & & & & & & \\
\hline Read span & .41 & .29 & - & .22 & - & & & & & \\
\hline Listen span & .48 & .32 & - & .19 & .46 & - & & & & \\
\hline Least span & .31 & .26 & - & .25 & .43 & .36 & - & & & \\
\hline VM & .74 & .31 & - & .16 & .50 & .48 & .44 & - & & \\
\hline Cross Out & .72 & .36 & - & .22 & .46 & .42 & .36 & .83 & - & \\
\hline PIAT Rec & .75 & .39 & - & .27 & .50 & .53 & .43 & .75 & .70 & - \\
\hline$M$ & 10.5 & 4.97 & - & 4.01 & 1.76 & 1.93 & 2.03 & 39.76 & 20.74 & 67.13 \\
\hline$S D$ & 1.6 & 1.07 & - & 0.77 & 0.61 & 0.79 & 0.66 & 8.54 & 5.19 & 17.44 \\
\hline
\end{tabular}

less than .10 indicate a good fit of the data to the model (Steiger, 1989).

The results of these analyses are shown in Table 3. In each study, the two-factor solution did not differ significantly from the data (i.e., produced nonsignificant values for $\chi^{2}$ ) and yielded acceptable values for the GFI and ARMS indices. In contrast, the one-factor solution differed significantly from the data in Study 1 and marginally so in Study 2. More informative, however, are comparisons of the relative fit of the one- and two-factor solutions. The difference in fit was significant in Studies 1 and $2\left[\chi^{2} \mathrm{~s}(1)=15.66\right.$ and 8.65 , respectively, $\left.p<.01\right]$. In other words, a one-factor model in which all memory tasks reflected the same underlying memory construct fit the data significantly less well than a two-factor model in which the memory tasks reflected two distinct memory constructs.

The best-fitting two-factor solution for each study is shown in Figure 1. One noteworthy feature of the results depicted in Figure 1 is the consistency across the two studies of the parameter estimates. For example, coefficients for the path from reading span to WM are .72 and .70 in Studies 1 and 2, respectively; coefficients for the path from listening span to WM are .63 and .64. Another important feature of these results is the finding that, although STM was distinguished from WM in both stud-

Table 2

Results of Exploratory Factor Analysis in Studies 1 and 2

\begin{tabular}{|c|c|c|c|c|c|c|}
\hline \multirow[b]{2}{*}{ Measure } & \multicolumn{2}{|c|}{ Entire sample } & \multicolumn{2}{|c|}{ 8- to 10 -year-olds } & \multicolumn{2}{|c|}{11 - to 13 -year-olds } \\
\hline & Factor 1 & Factor 2 & Factor 1 & Factor 2 & Factor 1 & Factor 2 \\
\hline \multicolumn{7}{|c|}{ Study 1} \\
\hline Letter span & .829 & .190 & .037 & .898 & .883 & .152 \\
\hline Word span & .854 & .130 & .344 & .721 & .884 & .004 \\
\hline Reading span & .203 & .817 & .856 & .120 & .176 & .785 \\
\hline Listening span & .117 & .855 & .795 & .186 & -.028 & .817 \\
\hline \multicolumn{7}{|c|}{ Study 2} \\
\hline Digit span & .275 & .743 & .443 & .646 & .029 & .828 \\
\hline Word span & .075 & .870 & -.043 & .889 & .220 & .792 \\
\hline Reading span & .809 & .125 & .833 & -.087 & .719 & .244 \\
\hline Listening span & .772 & .138 & .674 & .298 & .804 & -.100 \\
\hline Least number span & .706 & .202 & .728 & .156 & .654 & .224 \\
\hline
\end{tabular}

Note-There were 101 eight- to ten-year-olds and 54 eleven- to twelve-year-olds in Study 1 and 78 eight- to ten-year-olds and 64 eleven- to thirteen-year-olds in Study 2. 
Table 3

Results of Confirmatory Factor Analyses of Fit of Data to One- and Two-Factor Solutions

\begin{tabular}{cccc}
\hline & \multicolumn{3}{c}{ Measure of Fit } \\
\cline { 2 - 4 } Measure & $\chi^{2}$ & GFI & ARMS \\
\hline & Study 1 & & \\
One-factor solution & $15.66^{* *}$ & .9477 & .2278 \\
Two-factor solution & 0.00 & 1.00 & .0000 \\
& Study 2 & & \\
One-factor solution & $10.65 \dagger$ & .9707 & .0951 \\
Two-factor solution & 2.00 & .9943 & .0000 \\
\hline
\end{tabular}

Note-GFI denotes the Jöreskog-Sörbom goodness of fit index; ARMS denotes the adjusted root mean square. ${ }^{\dagger} p<.1 . \quad * * p<.01$.

ies, these constructs were not independent. Instead, they were correlated, by the same relatively modest amount in both studies.

Each sample included a fairly broad range of ages. To determine whether the two-factor solution held for both younger and older children, we divided the participants in the two studies into younger and older groups consisting of 8- to 10-year-olds and 11- to 13-year-olds, respectively. When the exploratory factor analyses were conducted for the younger and older samples in the two studies, a two-factor solution again emerged (see Table 2). In addition, when the confirmatory factor analyses were conducted for younger and older samples, in each case the fit of the one-factor solution was worse than the first of the two-factor solution [Study $1, \chi^{2} \mathrm{~s}(1)=6.16$ and 4.74 for younger and older children, respectively, $p<.05$; Study $2, \chi^{2} \mathrm{~s}(1)=3.88$ and 5.36 for younger and older children, respectively, $p<.05]$. Thus, throughout the age range studied here, the memory tasks distinguished STM and WM constructs.

\section{Links From Memory to Word Decoding Skill}

The next set of analyses were designed to further distinguish STM from WM by looking for distinctive relations between these memory constructs and word decoding skill. In these analyses, we compared three models: one in which both STM and WM were linked to decoding skill, one in which WM was linked to decoding skill but STM was not, and one in which STM was linked to decoding but WM was not. We used structural equation modeling (like that used in the confirmatory factor analysis) to assess these three models. As before, the focus of the analysis was the relative fit of the models that linked decoding with either WM or STM to the model in which decoding was linked to both WM and STM. In these analyses, the two-factor structure revealed in the confirmatory factor analysis was maintained by fixing the coefficients for the paths linking WM and STM to memory tasks.

The results of this analysis are shown in Table 4. In terms of absolute fit, the model in which decoding was linked to STM but not WM differed significantly from the observed pattern of correlations in Study 2 and marginally so in Study 1. The two other models-decoding linked to STM and WM and decoding linked only to WM-were both consistent with the data and had acceptable values on the GFI and ARMS measures.

However, the relative fits of the models show that WM is linked to decoding but STM is not. Specifically, relative to the model that linked both WM and STM to decoding, a model that linked only STM to decoding produced a significantly poorer fit to the data $\left[\chi^{2} \mathrm{~s}(1)=10.82\right.$ and 15.84, respectively, in Studies 1 and $2, p<.01]$. In contrast, relative to the model that linked both WM and STM to decoding, a model that linked only WM to decoding did not differ significantly in its fit to the data $\left[\chi^{2} \mathrm{~s}(1)=\right.$ 2.08 and 1.24, respectively, in Studies 1 and 2, $p>.1]$. The best-fitting model is shown in Figure 2.

Converging evidence for this conclusion comes from an analysis modeled after one used by Engle, Tuholski, et al. (1999). Specifically, as noted previously, WM and STM tasks have much shared variance. Factoring out this

\section{Study 1}

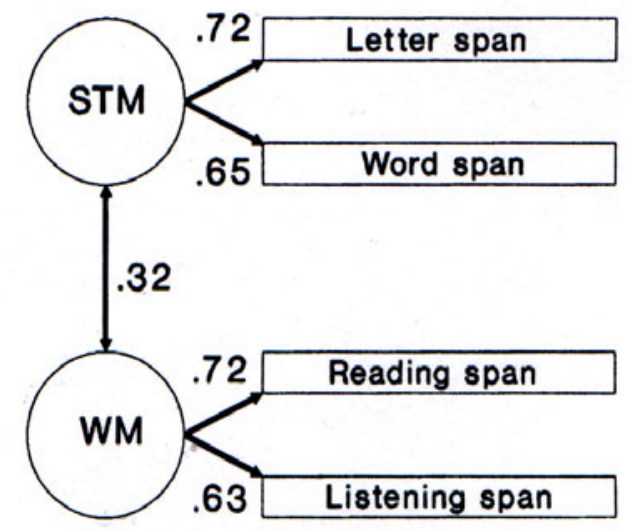

\section{Study 2}

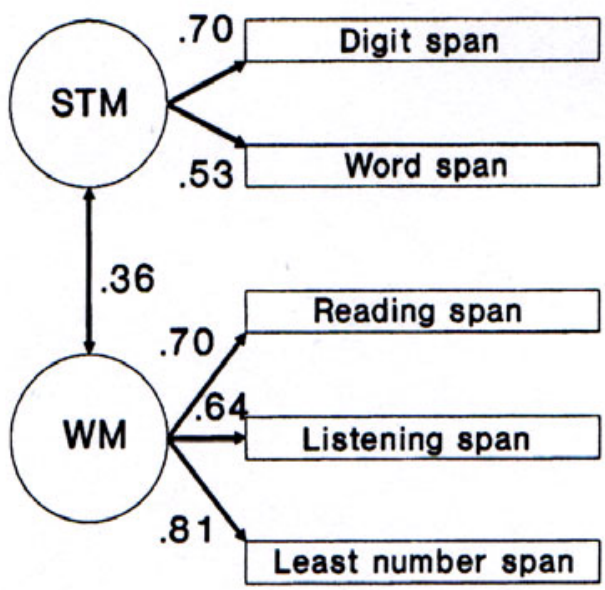

Figure 1. The best-fitting two-factor solution, shown separately for Studies 1 and 2. 
Table 4

Results of Confirmatory Factor Analyses of Links from Memory to Word Decoding Skill

\begin{tabular}{llll}
\hline \multirow{2}{*}{ Measure } & \multicolumn{3}{c}{ Measure of Fit } \\
\cline { 2 - 4 } & \multicolumn{1}{c}{$\chi^{2}$} & GFI & ARMS \\
\hline \multicolumn{4}{c}{ Study 1 } \\
WM and STM & 11.78 & .9738 & .0000 \\
Only STM & $22.60 \dagger$ & .9539 & .0377 \\
Only WM & 13.86 & .9699 & .0000 \\
& \multicolumn{1}{c}{ Study 2 } & \\
WM and STM & $28.30 \dagger$ & .9490 & .0095 \\
Only STM & $44.14 * *$ & .9293 & .0557 \\
Only WM & 29.54 & .9472 & .0000 \\
\hline
\end{tabular}

Note-GFI denotes the Jöreskog-Sörbom goodness of fit index; ARMS denotes the adjusted root mean square. $\quad t p<.1 . \quad * * p<.01$.

shared variance would leave only the variance that is unique to each construct. In terms of structural equation models, performance on all the memory tasks can be used to estimate the shared variance; with this variance removed, performance on the tasks thought to measure WM (or STM) estimates the variance unique to that construct. The critical outcome in this type of analysis is whether the coefficients for paths linking decoding skill to STM and WM are significant. In fact, the outcome was the same in both studies. The coefficients from WM to decoding - .41 and .53 in Studies 1 and 2, respectively-were significant $(t \mathrm{~s} \geq 3.77, p<.05)$, but those from STM to decoding-. 14 and .08 in Studies 1 and 2 , respectively-were not $(t \mathrm{~s} \leq 1.24)$. Thus, with the variance common to WM and STM factored out, WM was linked to decoding skill, but STM was not.

\section{Links From Processing Speed to Memory}

The final set of analyses concerned possible connections between memory and PS as measured by performance on the Visual Matching and Cross-Out tasks. In these analyses, we compared four models. In the initial model, PS was linked to both STM and WM. Three other models involved constraints on the initial model. In one, PS was linked equally to STM and WM (the path coefficients were forced to be the same); in a second, PS was linked to STM but not to WM; in a third, PS was linked to WM but not to STM. We used structural equation modeling to evaluate the absolute and relative fit of the models. As before, coefficients for paths from memory tasks to STM and WM were fixed at the values obtained in the confirmatory factor analysis.

The results of these analyses are shown in Table 5. Unlike the previous analyses in which the results were consistent across the two studies, in this instance Studies 1 and 2 produced different outcomes. Consequently, they are described for the two studies separately. In Study 1, all four models were consistent with the data (i.e., produced nonsignificant $\chi^{2} s$ ) and had acceptable values for the GFI and ARMS measures. However, relative to the initial model, a significantly poorer fit to the data was ob- tained by eliminating either the path from PS to STM or the path from PS to WM $\left[\chi^{2} \mathrm{~s}(1)=9.86\right.$ and $14.29, p<$ $.01]$. In contrast, the model in which PS was linked equally to STM and WM did not differ significantly from the initial model $\left[\chi^{2}(1)<1, p>.1\right]$.

In Study 2 , three of the four models were consistent with the data. The only model not consistent with the data was the one in which PS was linked to STM but not to WM. Compared to the initial model, the model linking PS to STM but not WM fit the data less well $\left[\chi^{2}(1)=\right.$ $33.76, p<.01]$, as did a model in which coefficients for paths from PS to STM and WM were the same $\left[\chi^{2}(1)=\right.$ $6.37, p>.05]$. In contrast, relative to the fit of the initial model, a model that linked PS to WM but not STM fit the data equally well $\left[\chi^{2}(1)=1.93, p>.1\right]$.

The best-fitting models for the two studies are shown in Figure 3. Study 1 yielded equal links from PS to STM and WM, but Study 2 resulted in a link from PS to WM only.

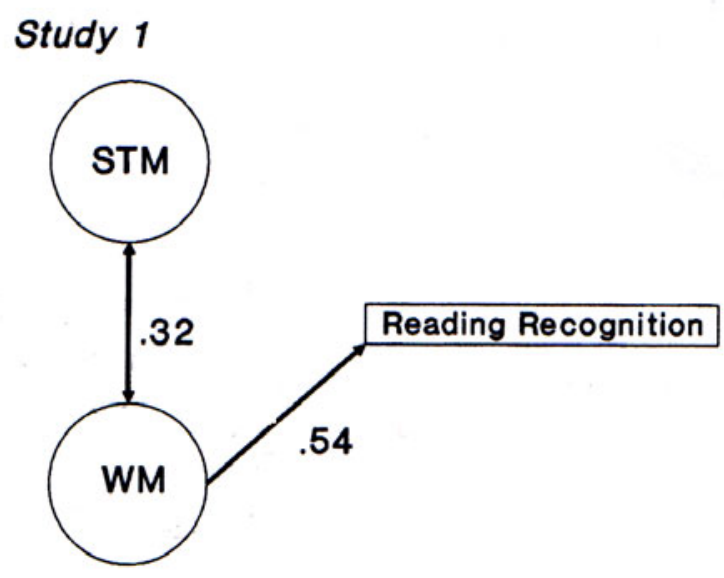

Study 2

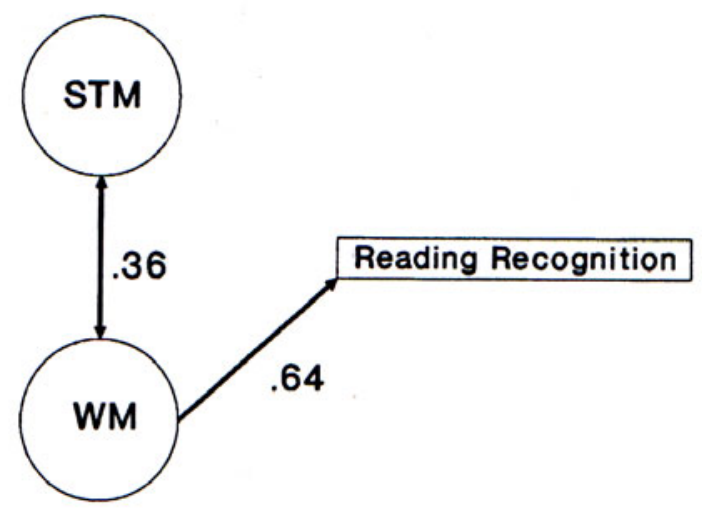

Figure 2. The best-fitting model that links word decoding (as measured by the Reading Recognition scale of the PIAT-R) to WM but not STM, shown separately for Studies 1 and 2 . 
Table 5

Results of Confirmatory Factor Analyses

of Links from Processing Speed to Memory

\begin{tabular}{lccc}
\hline & \multicolumn{3}{c}{ Measure of Fit } \\
\cline { 2 - 4 } \multicolumn{1}{c}{ Measure } & $\chi^{2}$ & GFI & ARMS \\
\hline & Study 1 & & \\
WM and STM & 9.17 & .9814 & .0000 \\
WM and STM equally & 9.34 & .9811 & .0000 \\
Only STM & 23.45 & .9535 & .0490 \\
Only WM & 19.03 & .9613 & .0256 \\
& Study 2 & & \\
WM and STM & 23.95 & .9576 & .0000 \\
WM and STM equally & 30.32 & .9462 & .0274 \\
Only STM & $57.71^{* *}$ & .9081 & .0891 \\
Only WM & 25.88 & .9538 & .0000 \\
\hline
\end{tabular}

Note-GFI denotes the Jöreskog-Sörbom goodness of fit index; ARMS denotes the adjusted root mean square. ${ }^{*} p<.05 . \quad{ }^{* *} p<.01$.

\section{DISCUSSION}

The primary results of this research were, first, that letter, digit, and word span tasks defined an STM factor, whereas reading, listening, and least number span tasks defined a WM factor; second, WM predicted word decoding skill but STM did not; and, third, links from PS to STM and WM were inconsistent across studies, with Study 1 showing equal links from PS to STM and WM but Study 2 showing a link from PS only to WM. In the remainder of this discussion, we comment on each of these findings.

\section{Structure of Memory Tasks}

As was the case in the study by Engle, Tuholski, et al. (1999), traditional span tasks loaded on one factor and complex span tasks (those requiring concurrent processing) loaded on a second factor. The present results extend those of Engle, Tuholski, et al. by expanding the number of simple and complex span tasks used to define the two factors. Whereas the STM factor in the Engle, Tuholski, et al. work was defined solely by word span tasks (forward and backward), in the present study this factor was defined by digit, letter, and word span tasks. Similarly, the WM factor in the Engle, Tuholski, et al. study was defined by reading span, operation span (in which participants remembered words while they solved simple math problems), and counting span (in which participants counted circles, then remembered the number of circles in a display). In the present study, the WM factor was defined by listening span and least number span tasks as well as reading span.

Expanding the catalogue of tasks in this manner is essential because factor analysis reveals relations between certain measures but does not provide an explanation for these relations. That is, strictly speaking, the present results and those of Engle, Tuholski, et al. (1999) simply show that traditional and complex span tasks load on separate factors; labeling these factors STM and WM is an inference based on theoretical analyses of the demands of the tasks. In the present case, simple span tasks are thought to assess memory traces that are active above threshold values; complex span tasks also measure such active traces as well as controlled processing that reflects the central executive. These inferences become more plausible as more tasks with these defining elements are shown to load differentially on the two factors.

The results also address Engle, Tuholski, et al.'s (1999) hypothesis regarding possible developmental change in STM and WM tasks. They argued that the rehearsal and chunking processes that contribute to accurate recall on STM tasks are probably less automated in children than in adults. Consequently, traditional span tasks may require more controlled processing with children than with adults, which would blur the distinction between STM and WM tasks at this stage in development. Contrary to this hypothesis, we found the same two-factor structure for younger and older children as that which Engle, Tuholski, et al. found for adults. Furthermore, the correlation between the two factors was actually smaller in the present studies $(\approx .3)$ than in Engle, Tuholski, et al. $(\approx .7)$. Although it is conceivable that Engle, Tuholski, et al.'s hypothesis might be supported with findings from even younger children, it is clear from the present studies that STM can be distinguished from WM by the elementary school years.

\section{Links Between Memory and Word Decoding}

The present studies confirm the finding reported previously by Swanson (1992) that WM was related to word decoding skill but STM was not. This relation probably represents two influences of WM on decoding, influences reflecting the storage and attentional functions of WM. On the one hand, many investigators (e.g., Gottardo, Stanovich, \& Siegel, 1996; Leather \& Henry, 1994) have reported positive correlations between decoding, complex span, and phonological awareness tasks in which participants pronounce words as they would sound with certain parts deleted (e.g., cat without /c/). One interpretation of this result is that individuals skilled at phonological coding are better able to retain information in the phonological loop of WM and thereby are better able to acquire and access letter-sound correspondences during reading.

The relation between WM and decoding must reflect more than phonological awareness. If it did not, decoding skill should have been predicted better by STM tasks than WM tasks, because the former provide arguably "purer" estimates of storage in the phonological loop. Given that WM did predict decoding skill more accurately than STM did, it appears that the attentional function of WM also contributes to the link with decoding. This argument has empirical support: Although much of the variance between WM and decoding is shared with phonological awareness, some is unique to WM (e.g., Gottardo et al., 1996). The argument also has theoretical support: For many elementary school children, decoding often requires controlled not automatic processing.

\section{Links From Processing Speed to Memory}

In both studies, PS was linked to WM. That is, more rapid processing was associated with more accurate re- 


\section{Study 1}

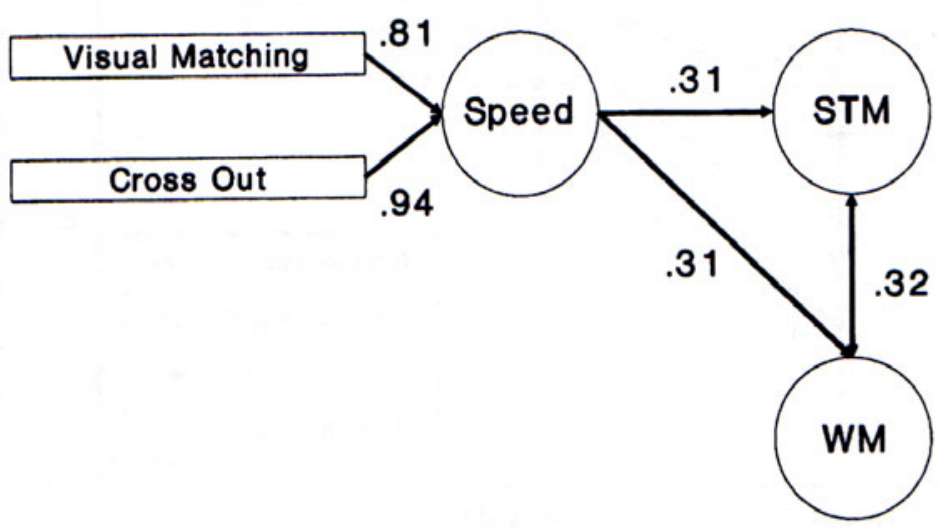

\section{Study 2}

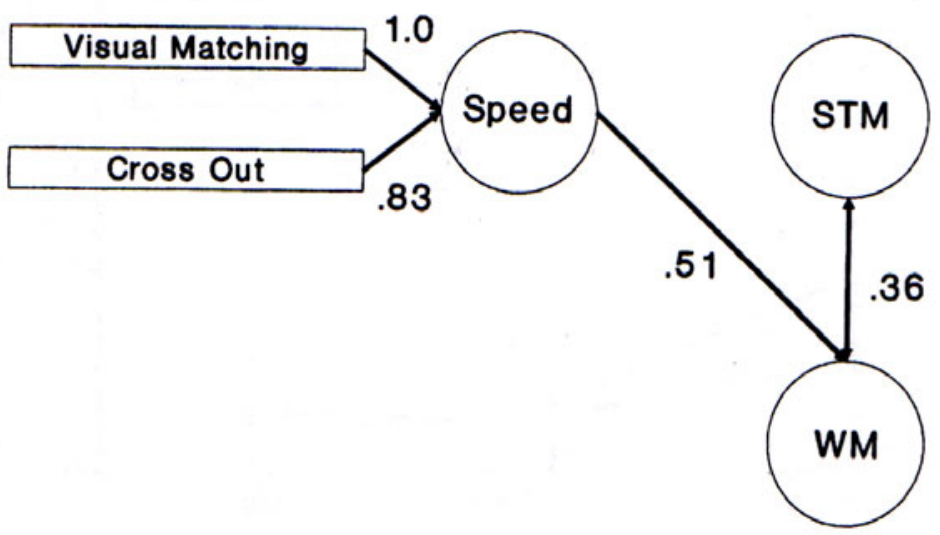

Figure 3. The best-fitting model that links PS to STM and WM, shown separately for Studies 1 and 2.

tention on complex span tasks. One account of this result is that increased PS results in more efficient controlled processing. That is, more rapid processing makes it possible to direct attention more readily between the competing demands of complex span tasks.

The results concerning PS and STM were inconsistent. Study 1 but not Study 2 indicated that PS was linked to STM. The STM and WM tasks differed somewhat in the two studies (e.g., letter span in Study 1 vs. digit span in Study 2), but even when one focuses on the tasks that were the same in the two studies, the outcomes of the studies differed. The two speeded tasks (Visual Match, CrossOut) were less strongly related to word span in Study 2 than in Study 1, but more strongly related to reading span and listening span in Study 2 than in Study 1. That is, correlations between PS and memory were much more differentiated in Study 2 than in Study 1. Given the many other consistencies across the two studies (e.g., in mean level of performance, in the pattern of other correlations), there is no obvious reason to identify one result as "true" and the other as "anomalous." Consequently, we suggest that additional data are needed to resolve this inconsistency before one tries to interpret possible links between PS and STM.

\section{REFERENCES}

Bachelder, B. L., \& Denny, M. R. (1977). A theory of intelligence: II. The role of span in a variety of intellectual tasks. Intelligence, $\mathbf{1}$, 237-256.

Cantor, J., Engle, R. W., \& Hamilton, G. (1991). Short-term memory, working memory, and verbal abilities: How do they relate? Intelligence, 15, 229-246.

Carpenter, P. A, Just, M. A., \& Shell, P. (1990). What one intelligence test measures: A theoretical account of the processing in the Raven Progressive Matrices Test. Psychological Review, 97, 404-431.

Cowan, N. (1988). Evolving conceptions of memory storage, selective attention, and their mutual constraints within the human information processing system. Psychological Bulletin, 104, 163-191.

Cowan, N. (1995). Attention and memory: An integrated framework. Oxford: Oxford University Press.

Cowan, N., Wood, N. L., Wood, P. K., Keller, T. A., Nugent, L. D., \& Keller, C. V. (1998). Two separate verbal processing rates con- 
tributing to short-term memory span. Journal of Experimental Psychology: General, 127, 141-160.

Daneman, M., \& CARPENTER, P. A. (1980). Individual differences in working memory and reading. Journal of Verbal Learning \& Verbal Behavior, 19, 450-466.

Engle, R. W., Kane, M. J., \& Tuholski, S. W. (1999). Individual differences in working memory capacity and what they tell us about controlled attention, general fluid intelligence, and functions of the prefrontal cortex. In A. Miyake \& P. Shah (Eds.), Models of working memory (pp. 102-134). Cambridge: Cambridge University Press.

Engle, R. W., Tuholski, S. W., Laughlin, J. E., \& Conway, A. R. A. (1999). Working memory, short-term memory, and general fluid intelligence: A latent-variable approach. Journal of Experimental Psychology: General, 128, 309-331.

Gottardo, A., Stanovich, K. E., \& Siegel, L. S. (1996). The relationships between phonological sensitivity, syntactic processing, and verbal working memory in the reading performance of third-grade children. Journal of Experimental Child Psychology, 63, 563-582.

KaIL, R, \& HALL, L. K. (1999). Sources of developmental change in children's word-problem performance. Journal of Educational Psychology, 91, 660-668.
KAIL, R., \& PARK, Y. (1994).Processing time, articulation time, and memory span. Journal of Experimental Child Psychology, 57, 281-291.

Kyllonen, P. C., \& Christal, R. E. (1990). Reasoning ability is (little more than) working-memory capacity? Intelligence, 14, 389-433. LeAther, C. V., \& Henry, L. A. (1994). Working memory span and phonological awareness tasks as predictors of early reading ability. Journal of Experimental Child Psychology, 58, 88-111.

Salthouse, T. A. (1996). General and specific speed mediation of adult age differences in memory. Journal of Gerontology, 51, P30P42.

Steiger, J. H. (1989). EzPATH: Causal modeling. Evanston, IL: SYSTAT.

SWANSON, H. L. (1992). Generality and modifiability of working memory among skilled and less skilled readers. Journal of Educational Psychology, 84, 473-488.

Towse, J. N., Hitch, G. J., \& Hutton, U. (1998). A reevaluation of working memory capacity in children. Journal of Memory \& Language, 39, 195-217.

(Manuscript received March 2, 2000; revision accepted for publication June 19, 2000.) 